\title{
Grundlegende Prozesse betreffend Hydraulik, Sedimenttransport und Flussmorphologie an der Donau
}

\author{
Philipp Gmeiner · Marcel Liedermann · Marlene Haimann · Michael Tritthart · Helmut Habersack \\ Online publiziert: 15. März 2016 \\ (C) Die Autor(en) 2016. Dieser Artikel ist auf Springerlink.com mit Open Access verfügbar.
}

Zusammenfassung Im Rahmen des Pilotprojektes Bad Deutsch-Altenburg wurde vor, während und nach der gleichzeitigen Umsetzung mehrerer Maßnahmen (Buhnenumbau, Granulometrische Sohlverbesserung - GSV, Uferrückbau und Gewässervernetzung) ein umfassendes Monitoringprogramm durchgeführt. Ziel war es, neben der Ist-Zustandserfassung, Begleitung und Dokumentation der Bauphase und der Erfassung der Entwicklung nach Bau auch die zugrunde liegenden Prozesse hinsichtlich Hydraulik, Sedimenttransport und Flussmorphologie $\mathrm{zu}$ analysieren. Erstmals wurde dabei die zeitliche Schwankung der Sohlschubspannung direkt gemessen. Die auftretenden Sohlschubspannungen folgten dabei in Abhängigkeit von der Turbulenzintensität einer Log-Normaloder einer Normalverteilung. Diese Schwankungen sind mit ein Grund, weshalb schon bei Niederwasser ein nennenswerter Geschiebetransport gemessen wurde. Der effektive Durchfluss des Geschiebetransportes liegt dabei im Bereich von 2000 und $2200 \mathrm{~m}^{3} / \mathrm{s}$. $50 \%$ Prozent des Geschiebes wird bei Durchflüssen bis $2300 \mathrm{~m}^{3} / \mathrm{s}$ transportiert, mit Jahresfrachten von 306.000 bis $594.000 \mathrm{t}$ für die Jahre 2006 bis 2014. Bei Betrachtung des Transportverhaltens einzelner Tracersteine in 3 Größenklassen durchwanderten $74 \%$

DI P. Gmeiner ( $\square)$ · DI Dr. M. Liedermann • DI M. Haimann · Priv.-Doz. DI .

Dr. M. Tritthart · Univ.-Prof. DI .

Dr. H. Habersack

Christian Doppler Labor für Innovative Methoden in Fließgewässermonitoring, Modellierung und Flussbau, Institut für Wasserwirtschaft, Hydrologie und konstruktiven Wasserbau, Department für Wasser-Atmosphäre-Umwelt, Universität für Bodenkultur Wien,

Muthgasse 107,

1190 Wien, Österreich

E-Mail: philipp.gmeiner@boku.ac.at der Steine die $3 \mathrm{~km}$ lange Strecke in einem Jahr, mit Geschwindigkeiten von 6,2 bis $10,6 \mathrm{~m} / \mathrm{d}$. Kleine Steine bewegten sich häufiger und weiter als mittlere und große, welche ein ähnliches Transportverhalten aufwiesen. Die erstmalig an der Donau beschriebenen Kiesdünen erreichten im Vergleich wesentlich höhere Geschwindigkeiten mit 2,5 bis $9 \mathrm{~m} / \mathrm{h}$. Ein Abgleich mit Fangkorbmessungen zeigte, dass Kiesdünen, wenn sie auftreten, den überwiegenden Teil des Geschiebetransportprozesses bestimmen.

Schlüsselwörter Geschiebetransport . Schwebstofftransport · Sohlschubspannung · Tracer · Sohlformen · Donau

Processes regarding hydraulics, sediment transport and river morphology at the Austrian Danube

Abstract In the scope of the Pilotproject Bad Deutsch-Altenburg a comprehensive monitoring program was conducted, prior, during and after the implementation of several measures (groyne reconstruction, granulometric bed improvement-GBI, river bank restoration and side arm reconnection). Beside the documentation of the current status, during the construction works and after the implementation of the measures, description and analyses of the underlying processes concerning hydrodynamics, sediment transport and morphology were a main objective. For the first time the instantaneous bed shear stress was measured directly in a gravel bed river. The probability distributions of the occurring bed shear stress, showed a dependency on the turbulence intensity, either following a log-normal or a normal distribution. Those fluctuations seem to be one of the reasons for a notable bed load transport, measured during low flow conditions in the Danube. The effective discharge lies in a discharge range from 2000 to $2200 \mathrm{~m}^{3} / \mathrm{s}$, with $50 \%$ of the bed load being transported at discharges up to $2300 \mathrm{~m}^{3} / \mathrm{s}$ and an annual bed load transport between 306,000 and 594,000 tons for the years 2006 to 2014 . Regarding the transport behaviour of single tracer stones $74 \%$ passed the $3 \mathrm{~km}$ long reach within one year, with a virtual velocity between 6,2 and $10,6 \mathrm{~m} / \mathrm{d}$. Small stones moved more frequent and farther, compared to the medium and big stones, who showed a comparable transport behaviour. In comparison gravel dunes moving with $2,5-9 \mathrm{~m} / \mathrm{h}$ have a substantially higher velocity, and tend to dominate the bed load transport process when occurring.

Keywords Bed load transport. Suspended sediment transport · Bed shear stress · Gravel tracer · Bed forms . Danube

\section{Einleitung}

Eingriffe in ein Flusssystem setzen ein gewisses Grundverständnis für die in ihm ablaufenden Prozesse und deren Interaktion voraus. $\mathrm{Zu}$ den klassischen flussbaulichen Herausforderungen zählen unter anderem Hochwasserschutz, Regulierungsmaßnahmen, Bau von Wasserkraftanlagen, Verbesserung der Schifffahrt, Rückbauprojekte bzw. Renaturierungen, Sedimentmanagement oder die Vorhersage morphologischer Entwicklungen. Obwohl der größte Masseanteil an Sediment auf Schwebstoffe entfällt, der unsere Landschaft verlässt (Milliman und Syvitski 1992), so ist es vor allem der Sedimenttransport gröberer Fraktionen, welcher die flussmorphologische Ausgestaltung in Kiesbettflüssen maßgeblich bestimmt. Im Gegensatz zu Reinwasserströmungen, welche durch die Kontinuums- und NavierStokes-Gleichungen hinreichend genau 
a

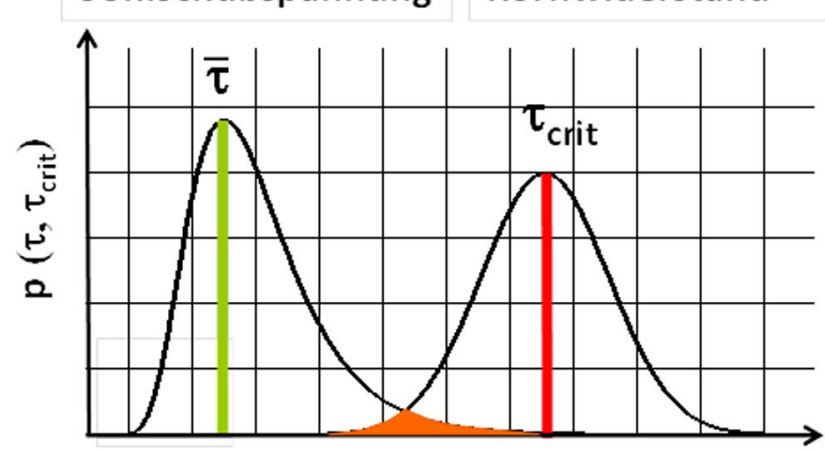

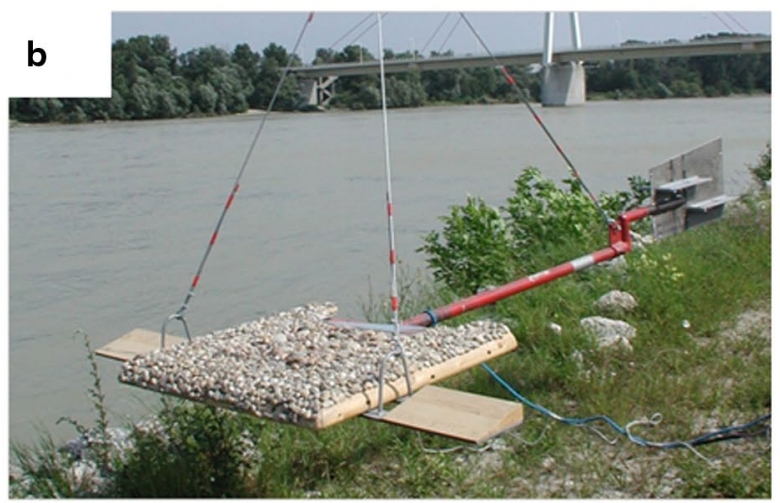

Abb. 1 a Dichtefunktion der Sohlschubspannung und des Kornwiderstandes. (Nach Grass 1970), b Messgerät zur direkten Erfassung der zeitlich schwankenden Sohlschubspannung

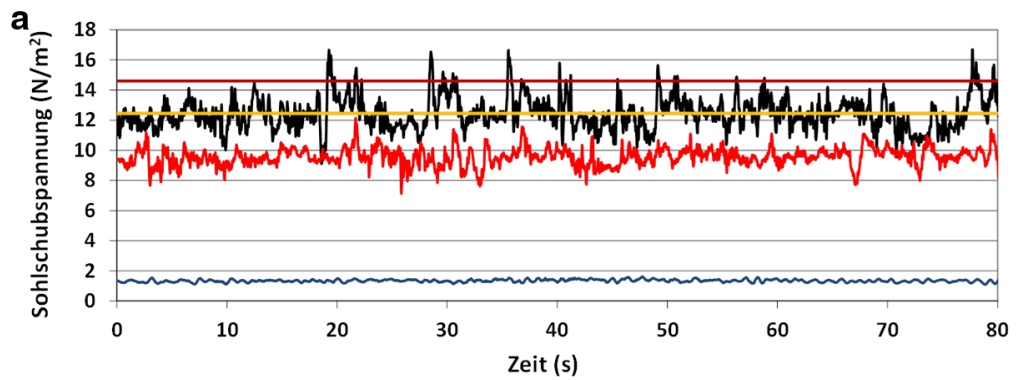

-Strommitte —Ufernah — Buhnenfeld - -Arithmetisches Mittel — tc $14.6 \mathrm{~N} / \mathrm{m}^{2}$ - Qc $1639 \mathrm{~m}^{3} / \mathrm{s}$

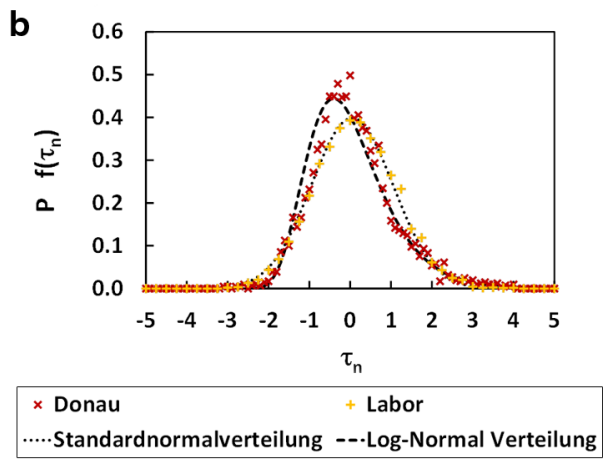

Abb. 2 a Zeitliche Schwankung der direkt gemessenen Sohlschubspannung in der Donau, b Häufigkeitsverteilung der auftretenden Sohlschubspannungen Vergleich Labor und Natur

beschrieben werden können, entziehen sich Sedimenttransportprozesse aufgrund ihrer Komplexität einer universelleren Beschreibung (Ferreira et al. 2015). Die ablaufenden Prozesse erstrecken sich dabei über einen großen Skalenbereich. Einzugsgebietsbezogen ist vor allem von Interesse, wo und in welchem Ausmaß Sediment verfügbar ist, und ob bzw. wie ein Transfer stromab möglich ist. Im Flussbett selber gibt es eine Vielzahl an möglichen Quellen und Senken für das transportierte Sediment. Seien es Schotterbänke, Kolke, natürliche oder künstlich geschaffene Hindernisse, um nur einige zu nennen. Betrachtet man den Sedimenttransport am Einzelkorn, so wird er von einer komplexen Fluid-Partikelund Partikel-Partikel- Interaktion bestimmt (Frey und Church 2011). Dieses Zusammenspiel zwischen turbulenter Strömung und kornbezogenen Prozessen wie hiding und exposure, mikrotopografischer Kornlagerung, Kornsortierung bzw. Einmischungsprozesse oder Sohlformen, führt zu einer räumlichen und zeitlichen Variabilität des Sediment- transportes und erschwert dessen genaue Abschätzung. Daher besteht mehr denn je die Notwendigkeit, Sedimenttransportmessungen durchzuführen, einerseits zur Schaffung einer entsprechenden Datengrundlage, andererseits um bestehende empirische Formelansätze zu erweitern und zu verbessern. Diese Daten werden auch für Entwicklung, Aufbau und Kalibrierung von Sedimenttransportmodellen benötigt, welche im Gegenzug unverzichtbare Werkzeuge für die Analyse der Auswirkungen flussbaulicher Maßnahmen darstellen. Denn diese bieten die Möglichkeit einer räumlichen und zeitlichen Skalierung (Inter- und/oder Extrapolation) von meist Punkt- oder Profilweise erfassten Messdaten.

Dieser Beitrag gibt eine Übersicht über die an Gewässersohle auftretenden Strömungskräfte und deren Einfluss auf Sedimenttransport sowie die daraus resultierenden Ergebnisse in Bezug auf Geschiebetransport, den vertikalen Aufbau der Donausohle und die Bedeutung von Hochwasserereignissen für den Schwebstofftransport.

\section{Innovationen und Erkenntnisse auf Prozessebene}

2.1. Direkte Messung und Analyse der Sohlschubspannung als Grundlage für den Sedimenttransport

Die zeitliche und räumliche Verteilung der Sohlschubspannung stellt ein wichtiges Bindeglied zwischen den turbulenten Strömungsverhältnissen und dem Sedimenttransport beziehungsweise dem Bewegungsbeginn des Geschiebes dar. Sie ist somit ein grundlegender Parameter für die Beschreibung und Vorhersage morphodynamischer Prozesse. Ausgehend von der Arbeit von Shields (1936) wird in der Regel ein zeitlich gemittelter Wert der Sohlschubspannung verwendet, um die an der Gerinneberandung auftretenden Kräfte zu beschreiben. Der deterministische Ansatz von Shields berücksichtigt jedoch nicht die zeitlichen und räumlichen Schwankungen der Sohlschubspannungen respektive der auftretenden Kräfte an der Gewässersohle, welche eng mit der Charakteristik 


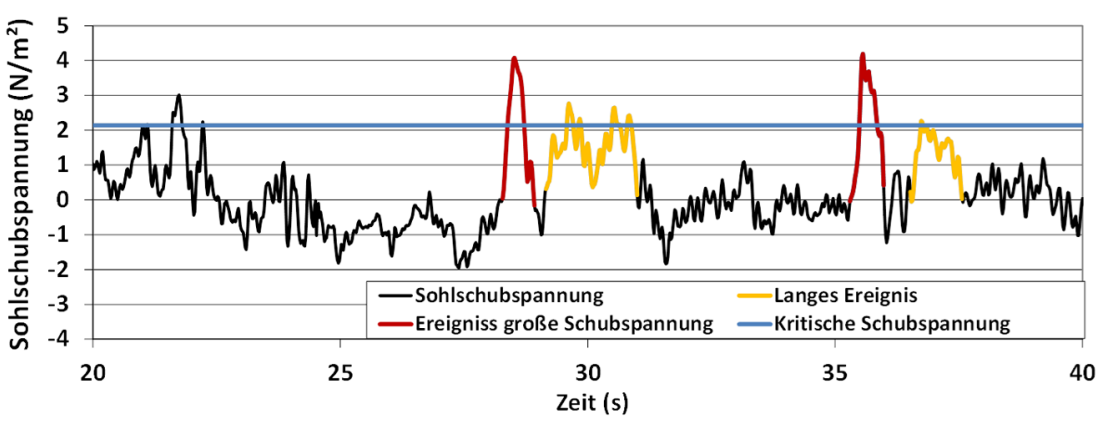

Abb. 3 Identifikation von Ereignissen mit maximaler Kraft bzw. mit langer Dauer in den direkt gemessenen Sohlschubspannungen (Bereich Strommitte)

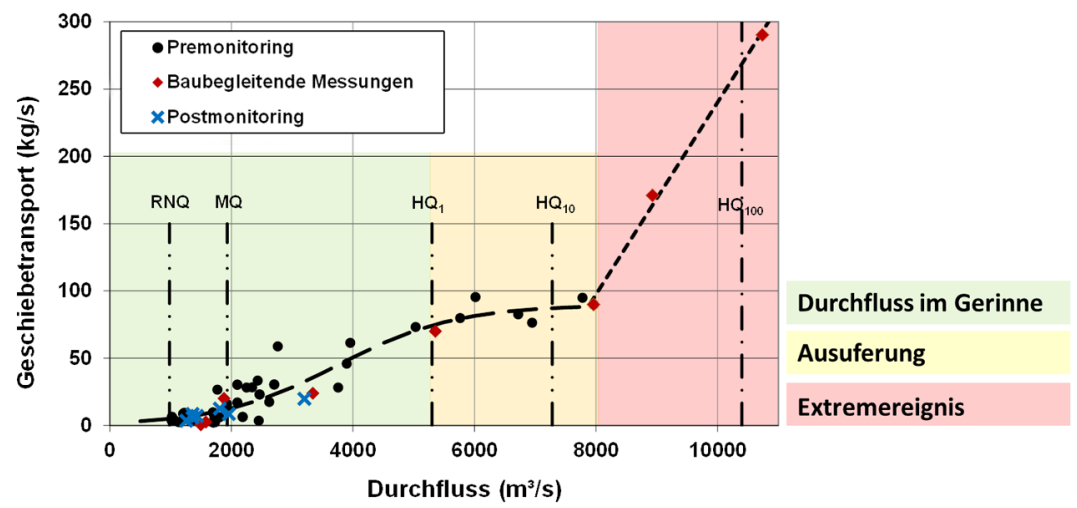

Abb. 4 Geschiebetransportcharakteristik im Bereich Bad Deutsch-Altenburg: Geschiebetransport im Querprofil in Abhängigkeit vom Donaudurchfluss

turbulenter Strömungen verbunden sind. Probabilistische Modelle scheinen die zugrunde liegenden Prozesse wesentlich besser zu beschreiben (Einstein und El-Samni 1949; Grass 1970; McEwan und Heald 2001; Ancey und Heyman 2014). Diese statistischen Modelle beschreiben die Strömungskräfte sowie die kritische Schubspannung als Dichtefunktionen und nicht anhand von einzelnen Werten (McEwan und Heald 2001). Durch die Überlagerung der Dichtefunktionen von Sohlschubspannung und Kornwiderstand erhält man die Bewegungswahrscheinlichkeit eines Sedimentkornes (Abb. 1a).

Es gibt zwar eine Vielzahl an indirekten Methoden zur Berechnung der Sohlschubspannung (über den Gleichgewichtsansatz, das logarithmische Geschwindigkeitsprofil,

die Reynoldsspannungen oder die turbulente kinetische Energie), sie erlauben jedoch nur die Abschätzung einer zeitlich gemittelten Sohlschubspannung. Um die zeitliche Schwankung der Sohlschubspannung $\mathrm{zu}$ erfassen, wurde sie mit tät (Verhältnis Standardabweichung zu arithmetischem Mittelwert) einer LogNormal-Verteilung (Strommitte) folgt beziehungsweise sich in Ufernähe sowie im Buhnenfeld oder auch im Labor immer mehr einer Normalverteilung annähert. Dies bedeutet, dass in Bereichen mit einer Log-Normal-Verteilung größere Schubspannungswerte häufiger auftreten, als im Vergleich zu Bereichen, die einer Normalverteilung folgen. Laut Diplas et al. (2008) ist es jedoch nicht ausreichend, nur die Größe und Verteilung der Schwankung zu betrachten, sondern auch den Zeitraum über den die Kraft wirkt - Impulskonzept: Kombination von Kraft und Zeit. Daher wurde der Impuls (hier Kraft mal Zeit) von Ereignissen mit großer Stärke bzw. von langer Dauer berechnet (Abb. 3).

Es zeigt sich dabei, dass durchaus Ereignisse auftreten, welche lange genug andauern, um den gleichen Impuls auf ein Sedimentkorn auszuüben wie Ereignisse bei maximaler Schubspannung. Dies verdeutlicht, dass es notwendig scheint, für die Beschreibung des Sedimenttransportes nicht nur die auftretenden Kräfte, sondern auch deren Dauer zu berücksichtigen.

\subsection{Bewegungsbeginn, Geschiebetransport, -fracht}

In der Projektstrecke (Donau Strom-km 1887,5 bis 1884,5 ) wurde erstmals an der österreichischen Donau eine längere Reihe von Geschiebemessungen durchgeführt, die nun Durchflüsse bis $\mathrm{zu}$ einem $\mathrm{HQ}_{200}$ abdecken. Durch die nun zur Verfügung stehende Messreihe konnten Erkenntnisse bezüglich des Geschiebetransportes an der Donau gewonnen werden. So konnte schon bei Messungen um Regulierungsniederwasser $\left(980 \mathrm{~m}^{3} / \mathrm{s}-\mathrm{KWD} 2010\right)$ nennenswerter Transport beobachtet werden (z. B. waren Tracersteine der Korngröße $27,5 \mathrm{~mm}, 40$ und $70 \mathrm{~mm}$ in Bewegung und bei Geschiebemessungen wurden Körner größer $40 \mathrm{~mm}$ erfasst, ebenso waren Steine der Größe 40 bis $70 \mathrm{~mm}$ auf Videoaufnahmen in Bewegung zu erkennen). Ein weiteres Charakteristikum konnte bei Durchflüssen ab dem bordvollen Durchfluss beobachtet werden: Hier steigt der Geschiebetransport bis zur völligen Flutung der Vorländer kaum weiter an (Gelber Bereich in Abb. 4). Erst bei Extremereignissen (Roter Bereich in Abb. 4) kann wieder ein deutlicher Anstieg verzeichnet werden. 


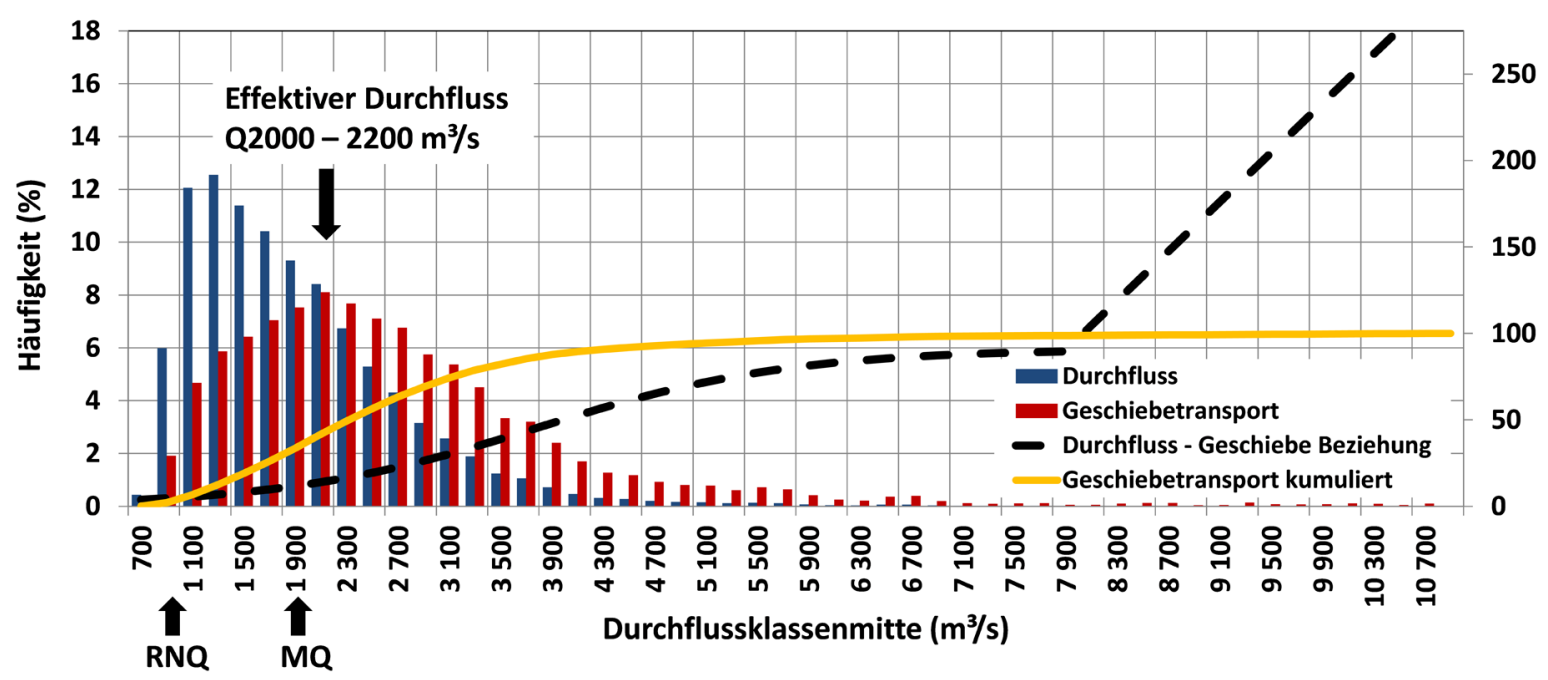

Abb. 5 Durchfluss-Geschiebetransportbeziehung, Auftrittshäufigkeit von Durchfluss und Geschiebetransport, sowie effektiver Durchfluss des Geschiebetransports der Donau im Bereich Bad Deutsch-Altenburg

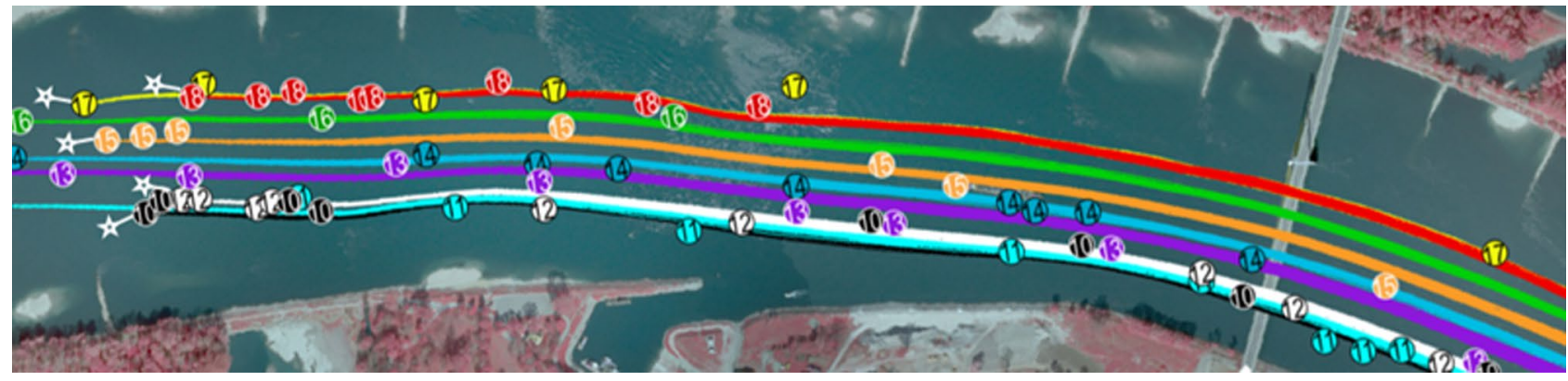

Abb. 6 Modellierte Tracerpfade (farbige Pfadscharen) im Vergleich zu den beobachteten Tracerpositionen (farbige Kreise); die Startpositionen der Tracersteine sind mit einem Stern markiert. (Modifiziert aus Liedermann et al. 2013)

Die52bisherigenGeschiebemessungen im Querprofil wurden mit dem Donaudurchfluss in Beziehung gesetzt und eine mögliche Variante für eine Ausgleichsfunktion ermittelt (Abb. 4). Mithilfe der Beziehung zwischen Durchfluss und Geschiebetransport (2006-2015) und einer langen Reihe an Durchflussdaten (1977-2015) kann durch die Auftrittshäufigkeit ein effektiver Durchfluss bzw. ein Durchflussbereich bestimmt werden, bei dem insgesamt gesehen der größte Geschiebetransport auftritt. Wie in Abb. 5 $\mathrm{zu}$ sehen, liegt der effektive Durchfluss zwischen 2000 und $2200 \mathrm{~m}^{3} / \mathrm{s}$ (Mittelwasser $1930 \mathrm{~m}^{3} / \mathrm{s}$ bis sommerliches Mittelwasser $2230 \mathrm{~m}^{3} / \mathrm{s}$ ).

Im Vergleich $\mathrm{zu}$ anderen Flüssen (z. B. die Drau bei Dellach mit einem effektiven Durchfluss knapp unter einem $\mathrm{HQ}_{1}$ ), liegt der Durchflussbereich, der am meisten zum Geschiebetransport beiträgt, an der Donau sehr tief. Dies äußert sich auch darin, dass im Durch- flussbereich zwischen RNQ (980 $\left.\mathrm{m}^{3} / \mathrm{s}\right)$ und MQ $\left(1930 \mathrm{~m}^{3} / \mathrm{s}\right)$, bereits $\sim 35 \%$ des Geschiebetransportes stattfinden. $50 \%$ des Geschiebes werden von Durchflüssen bis $2300 \mathrm{~m}^{3} / \mathrm{s}$ transportiert und $95 \%$ der Jahresfracht entfallen auf Durchflüsse bis $5300 \mathrm{~m}^{3} / \mathrm{s}$. Die jährliche Geschiebefracht in den Jahren 2006 bis 2014 bewegt sich dabei zwischen 306.000 und $594.000 \mathrm{t}$ oder einem Volumen von 196.000 bis $381.000 \mathrm{~m}^{3}$.

\subsection{Beobachtung des \\ Transportverhaltens einzelner Steine}

Neben der im vorangegangenen Kapitel beschriebenen Messung des Geschiebedurchgangs an einem Punkt (Euler'sche Betrachtungsweise) ist die gezielte Beobachtung der Einzelbewegungen von Steinen (Lagrange'sche Betrachtungsweise) eine ergänzende Methode, um weitere Einblicke in den Prozess des Geschiebetransports $\mathrm{zu}$ erhalten. $\mathrm{Zu}$ nennen wären der Bewegungsbeginn bzw. die Bewegungswahrscheinlichkeit, korngrößenabhängige Unterschiede im Transportverhalten, den Transportwegen, -geschwindigkeiten und -distanzen, sowie die Dauer von Ruhephasen bzw. Bewegungsphasen. Tracerlangzeitstudien (über mehrere Niederwasserund Hochwasserperioden hinweg) ermöglichen auch eine ganzheitliche Betrachtung des Geschiebetransportes, da zeitliche Schwankungen der Strömungsgrößen und räumliche Heterogenität an der Flusssohle Eingang in die gewonnenen Datensätze finden. Darüber hinaus erwiesen sich die Tracersteine auch als sehr wertvolles Werkzeug, um kurzfristig die Stabilität der GSV zu beurteilen (Liedermann et al. 2016, in dieser Ausgabe). Dazu wurde ein für einen Fluss von der Dimension der Donau einmaliges intensives Monitoring 

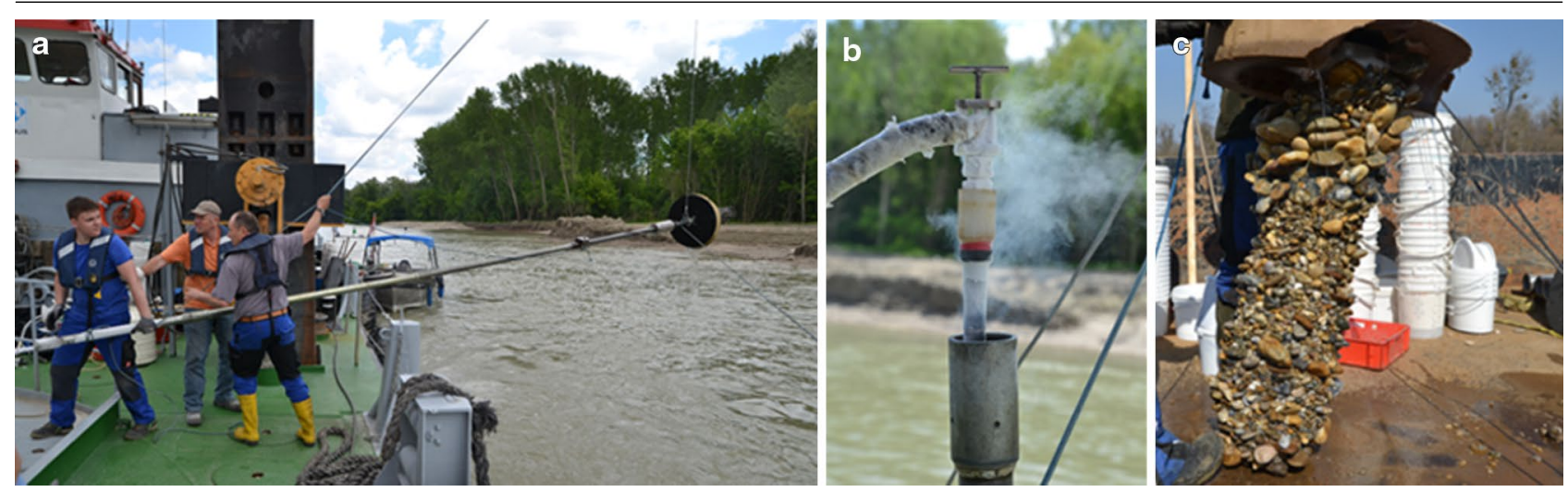

Abb. 7 a Einbringen des Freeze-Core-Gestänges, b Gefrieren der Donausohle mit Flüssigstickstoff, c Freeze-Core-Probe mit GSV-Schicht

von 40 Radiotracern (3 Größen mit einer b-Achse von 27,5, 40 und $70 \mathrm{~mm}$ ) vor den Umbaumaßnahmen durchgeführt. Über den Zeitraum von mehr als einem Jahr wurden die Tracersteine einmal wöchentlich gesucht und verortet. $74 \%$ der Steine durchwanderten die $3 \mathrm{~km}$ lange Strecke innerhalb eines Jahres. Die zurückgelegten Pfade waren dabei mehrheitlich uferparallel (Abb. 6), der Anteil des Quertransportes am Geschiebetransport lag bei $4 \%$ des longitudinalen Transportes und wird hauptsächlich von der Morphologie beeinflusst.

Die gemessene Transportwahrscheinlichkeit und -geschwindigkeit für die kleinen Steine (b-Achse 27,5 mm) lag bei $42 \%$ und 10,6 m/d. Die mittleren und großen Steine (b-Achse 40 und $70 \mathrm{~mm}$ ) hatten eine Transportwahrscheinlichkeit von 30,4 bzw. $26,1 \%$ bei einer Geschwindigkeit von 7,7 bzw. 6,2 m/d. Die kleineren Steine bewegten sich somit häufiger und weiter als die mittleren und großen, welche ein ähnliches Transportverhalten aufwiesen. Bei den während des baubegleitenden Monitorings eingebrachten Tracersteinen reduziert sich die Geschwindigkeit der mittleren Steine (40 mm) um zirka die Hälfte auf 3,2 m/d und die der großen auf zirka ein Drittel $(1,9 \mathrm{~m} / \mathrm{d})$, bei gleichzeitiger Reduktion der Transportereignisse. Ein Tracerstein gleicher Größe bewegt sich direkt nach den Baumaßnahmen daher etwas seltener und deutlich weniger weit als ein Stein während des Premonitorings. Die Daten der 40 Tracersteine aus dem Premonitoring wurden auch für die Kalibrierung eines numerischen Tracermodelles verwendet (Tritthart et al. 2012). Unter der Annahme einer konstanten Steinbewegung und eines Widerstandbeiwertes (Kalibrierparameter) sowie unter Einbeziehung des lokalen Sohlgradienten und einer variablen Schubspannungsgeschwindigkeit (generiert aus einer Gauß'schen Verteilung) wurden die Pfade und Geschwindigkeiten der Tracersteine für unterschiedliche Durchflüsse erfolgreich reproduziert. Dies schafft die Möglichkeit der räumlichen und zeitlichen Hochskalierung vorhandener Tracerbeobachtungen; somit ist es möglich, eine im Vergleich zu Naturmessungen große Anzahl an Steinbewegungen und deren Ausbreitungsverhalten für unterschiedliche Durchflussbereiche abzubilden.

\subsection{Die vertikale Entwicklung der Donausohle}

Eine wesentliche Grundlage zur Beurteilung des Einmischverhaltens bzw. der Stabilität und des Vorhandenseins der GSV ist eine detaillierte Kenntnis des Sohlaufbaus (Korngröße, Schichtungen, Reifegrad der Deckschicht etc.). Darüber hinaus dient der Sohlaufbau (unterteilt in 10-cm-Schichten) auch als Eingangsdatensatz für die Sedimenttransportmodellierung. Die Entnahme von Freeze Cores aus der Gewässersohle bietet dabei eine einzigartige Möglichkeit, eine ungestörte Probe der Donausohle bis in eine Tiefe von 1 bis $1,5 \mathrm{~m}$ zu ziehen. Dazu wird ein Stahlrohr in die Flusssohle geschlagen und anschließend mit Flüssigstickstoff heruntergekühlt, sodass das Sediment festfriert und ungestört entnommen werden kann (Abb. 7).

In Abb. 8 sind Freeze Cores mit unterschiedlichen GSV-Körnungen bei variablem Einmischungsgrad und einer klar erkennbaren Sohlschichtung dargestellt. Eine noch zur Gänze vorhandene GSV-Schicht der Körnung 40/70 ist in Abb. 8a zu sehen. Sie weist eine Schicht- dicke von $30 \mathrm{~cm}$ (Einbaustärke) auf und ist rund $10 \mathrm{~cm}$ von Normalgeschiebe überlagert. Eine eingemischte und nur noch teilweise vorhandene GSV-Schicht der Körnung 32/132 ist in Abb. 8b zu sehen. Diese Probe wurde in einem Bereich mit höherer Strömungsbelastung bei Strom-km 1884,9 genommen.

Die in Abb. 8c dargestellte FreezeCore-Probe entstammt dem Furt-Bereich bei Strom-km 1886,62. An ihr zeigt sich ausgehend vom Fremdgeschiebe (geologischer Tracer Körnung 40/70) ein dreiteiliger Aufbau der Donausohle, mit einem $d_{m}$ der zwischen 18,03 und $34,83 \mathrm{~mm}$ schwankt. Über die Kombination von Siebanalysen und visueller Beurteilung war es möglich, den Anteil der GSV am jeweiligen Freeze Core über die Tiefe darzustellen (Abb. 9).

Die in Abb. 10 dargestellten Freeze Cores wurden 4 Wochen und $1 \mathrm{Jahr}$ nach dem Einbau des Materials gezogen. Es handelt sich um GSV-Material, welches noch nicht überdeckt ist und somit direkt dem Strömungsangriff ausgesetzt ist.

Auffällig ist der offene Schotterlückenraum, welcher im Vergleich zu überdeckten Stellen nicht durch Perkolation oder kinetische Siebung mit Sand oder Feinkies verfüllt wurde.

Da sich das zugegebene GSV-Material innerhalb der Bandbreite des natürlich vorkommenden Sohlmaterials bewegt, ist die Identifikation des GSV-Materials nach erfolgter Durchmischung nur noch bedingt möglich. Die vorher aufgebrachte klar erkennbare Schicht von $30 \mathrm{~cm}$ verliert ihre Identität und die Vergröberung der Sohle kann sich immer noch innerhalb einer natürlich auftretenden Variabilität bewegen. Deshalb wurde für eine genauere Beobachtung des Einmischverhaltens bzw. der Einmischtiefe sowie von Translation und Diffusion der Steine 

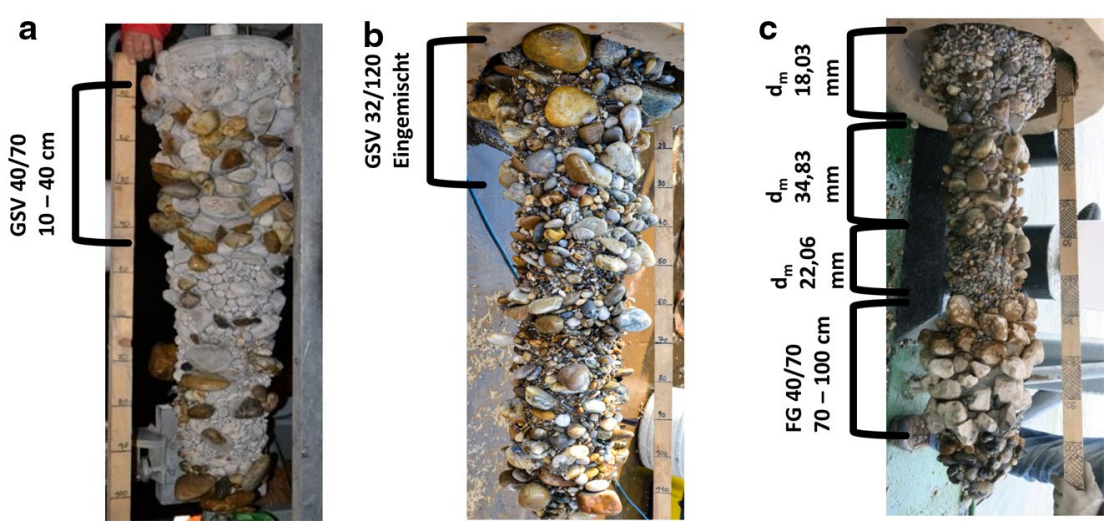

Abb. 8 Beispiele unterschiedlicher GSV-Schichten und Einmischungsgrade: a GSVSchicht Körnung 40/70, Lage 10-40 cm unter Gewässersohle, b GSV-Körnung 32/120, eingemischt in die Donausohle, c Beispiel für das Auftreten von Schichten mit unterschiedlichem $d_{m}$ bei Strom-km 1886,62 - Schicht 70-100 cm Fremdgeschiebe (geologischer Tracer)

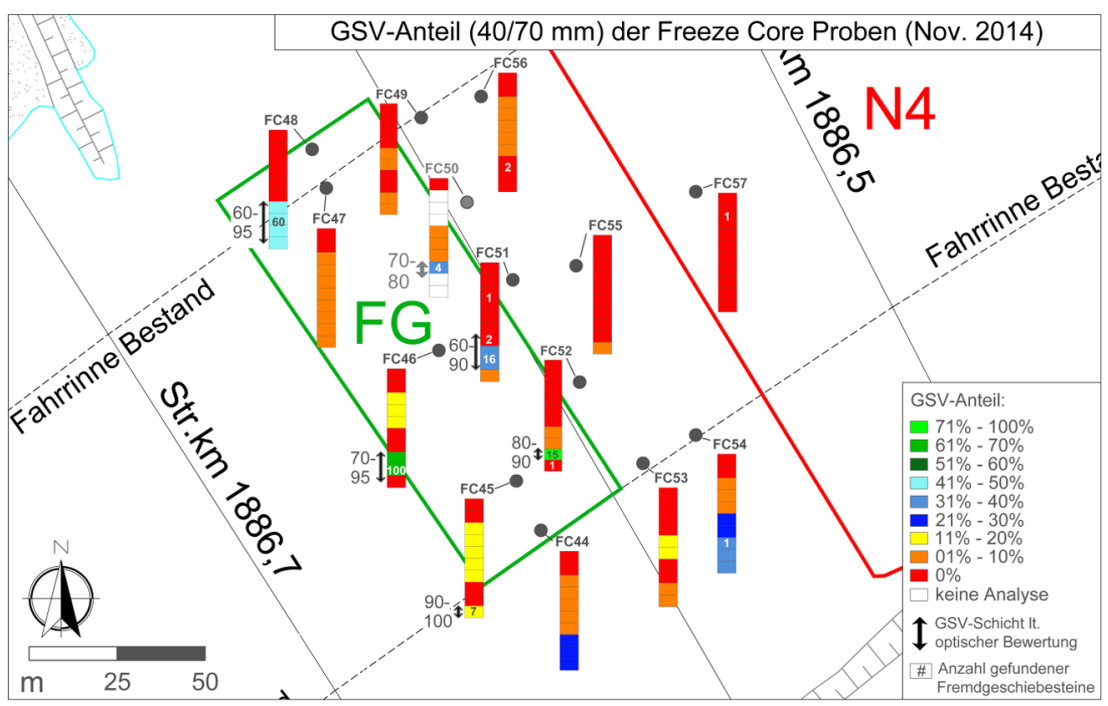

Abb. 9 Überblick über den GSV-Anteil (berechnet und visuell beurteilt) der FreezeCore-Proben am Probenahmeort
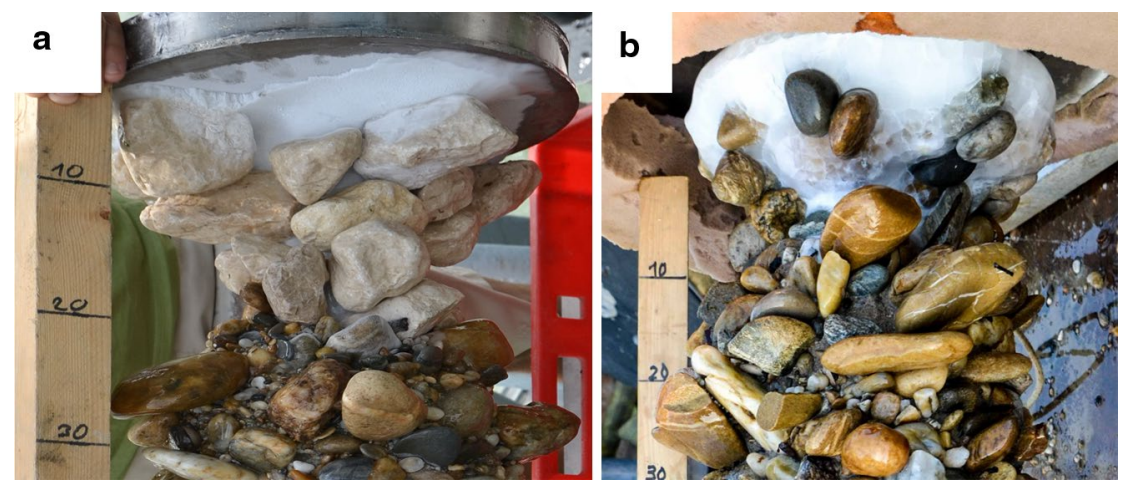

Abb. 10 a geologischer Tracer (Fremdgeschiebe) 4 Wochen nach Einbau, b GSV 32/120 rund 1 Jahr nach Einbau ein Streifen von 120 auf $60 \mathrm{~m}$ mit petrografisch und vor allem visuell klar abgrenzbaren geologischen Tracersteinen (Abb. 10a) belegt.

\subsection{Sohlformen und deren Einfluss auf den Geschiebetransport an der Donau}

Ein weiteres bisher unerforschtes bzw. sogar unbekanntes Charakteristikum der Donau sind die Sohlformen. Diese wurden durch hochauflösende Sohlgrundaufnahmen mittels Multi Beam entdeckt und wurden wegen deren großer Auswirkung auf den Bauablauf genauer analysiert. Grundsätzlich sind Kiesdünen auch in der internationalen Literatur kaum beschrieben, weshalb deren Charakteristik an der Donau auch gänzliches Neuland darstellt. Zuerst wurden die Dünenformen mithilfe von mehreren Multi-Beam-Aufnahmen zu unterschiedlichen hydrologischen Bedingungen analysiert (Abb. 11). Die durchschnittlichen Dünenlängen schwanken zwischen 7,8 und 10,7 $\mathrm{m}$, die Dünenhöhen schwanken zwischen 10 und $45 \mathrm{~cm}$ (Liedermann et al. 2014).

Die auftretenden Längen der Dünen zeigen keine sichtbare Abhängigkeit vom Durchfluss (Abb. 11a), bei den Dünenhöhen hingegen ist eine Zunahme mit steigendem Durchfluss feststellbar (Abb. 11b).

Während der Bauarbeiten wurde offensichtlich, dass vorbereitete Einbaufelder für die granulometrische Sohlverbesserung (GSV) oft nach wenigen Stunden wieder zusedimentiert waren. Daher wurde ein zeitlich hochaufgelöstes Monitoringprogramm durchgeführt, um die Dünenbewegung zu analysieren. So wurden alle 10 Minuten über mehrere Stunden hinweg Sohlgrundaufnahmen eines Teilbereiches der Stromsohle gemacht. Durch Vergleich der gewonnenen Sohlmorphologien konnte eine Sohlformgeschwindigkeit bestimmt werden, die zwischen $2,5 \mathrm{~m} / \mathrm{h}$ und $9 \mathrm{~m} / \mathrm{h}$ liegt und einen Mittelwert bei $5,6 \mathrm{~m} / \mathrm{h}$ besitzt (Bezug Messreihe Fa. Strabag, bei Einbeziehung der Messungen via donau: 5,26 m/h) (Abb. 12). Über die charakteristischen Formen der Dünen konnte ein Sedimentvolumen über eine längere Zeit ermittelt werden, um auf eine Geschiebetransportrate basierend auf dem Sohlformtransport schließen zu können. Für diesen spezifischen Geschiebetransport konnten Werte zwischen $0,20 \mathrm{~kg} / \mathrm{s} \mathrm{m}$ und $0,76 \mathrm{~kg} / \mathrm{s} \mathrm{m}$ 

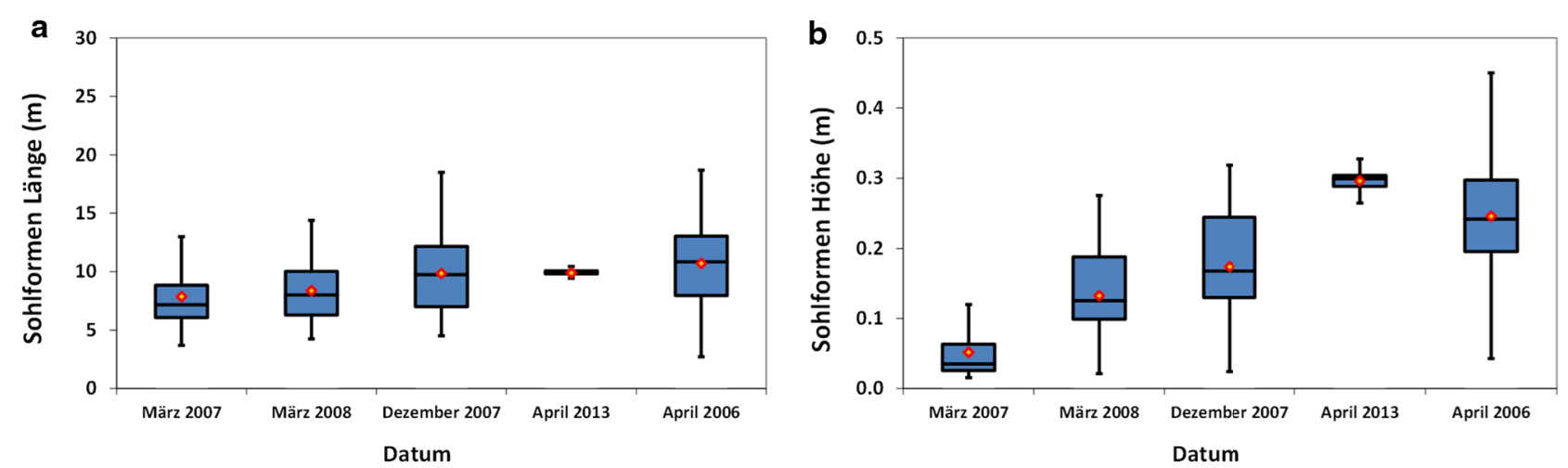

Abb. 11 Ausbildung von Sohlformen in Abhängigkeit vom auftretenden Durchfluss: a Beziehung zwischen Sohlformenlänge und Durchfluss, b Zusammenhang zwischen Sohlformenhöhe und Durchfluss
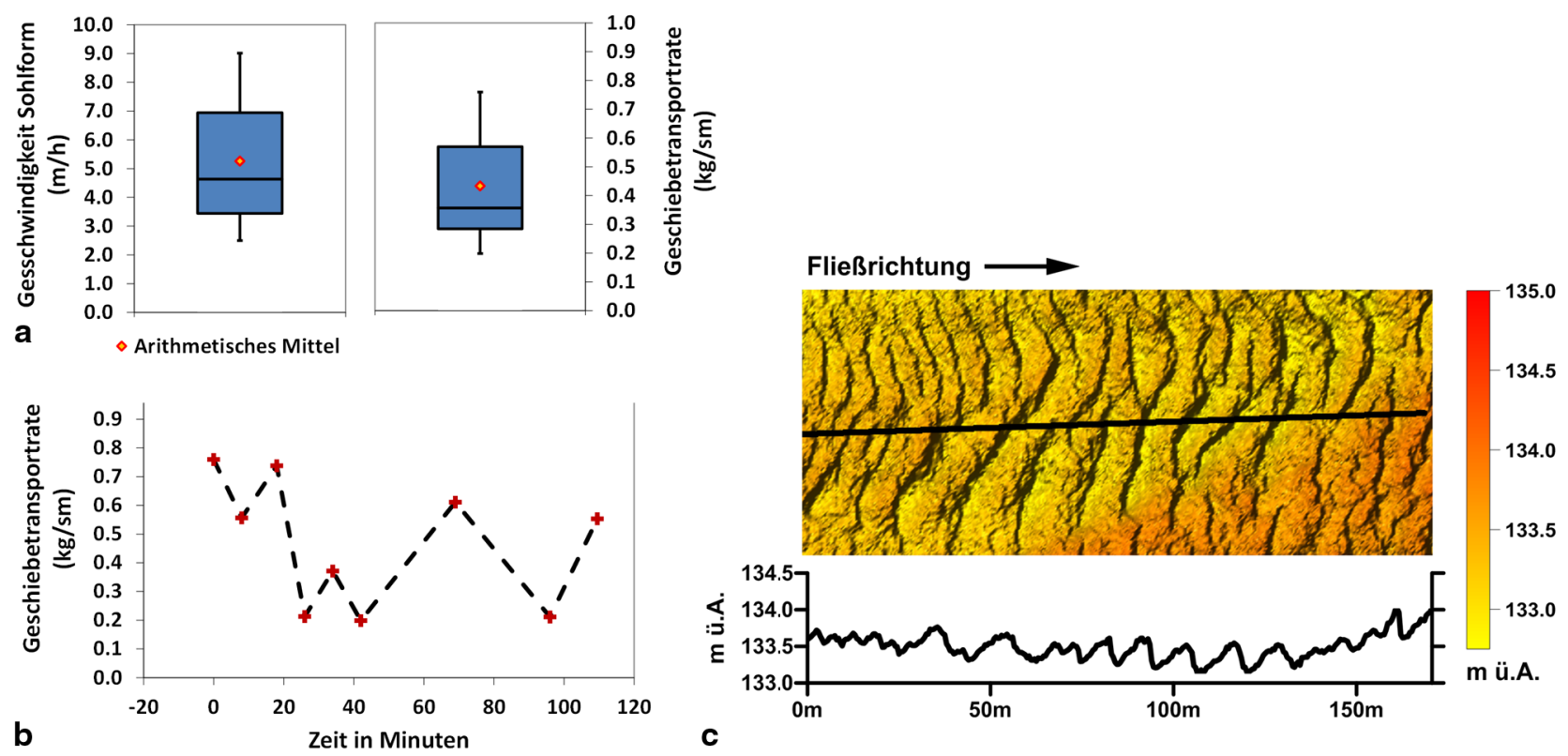

Abb. 12 Analyse der Sohlformen: a Geschwindigkeit und Geschiebetransportrate der betrachteten Sohlformen, b zeitliche Schwankung der Geschiebetransportrate zwischen sukzessiven Aufnahmen, c Sohlformen in Draufsicht und Schnitt (Schnitt 20-fach überhöht)

bei einem Mittelwert von $0,43 \mathrm{~kg} / \mathrm{s} \mathrm{m}$ gefunden werden (Liedermann et al. 2014).

Diese Ergebnisse passen gut zu den mittels Fangkorbmessungen gewonnenen Werten, weshalb davon ausgegangen werden kann, dass bei Auftreten von Kiesdünen der Geschiebetransport an der Donau fast ausschließlich von diesen bestimmt wird.

\subsection{Schwebstofftransportprozesse}

Es hat sich gezeigt, dass die korrekte Berechnung von Schwebstofffrachten während Hochwasserereignissen entscheidend ist, um zuverlässige Ergeb- nisse für die Jahresfrachten zu erhalten. Wie die Messungen der letzten Jahre (v. a. auch bei Hochwässern) ergaben, kann man jedoch nicht davon ausgehen, dass selbst bei Hochwässern eine vollständige und gleichmäßige Durchmischung der Schwebstoffe im Profil auftritt (Haimann et al. 2014). Bei vollständiger Durchmischung müsste sich der Querprofilbeiwert $\mathrm{k}_{\mathrm{p}}$ (Verhältnis mittlerer $\mathrm{zu}$ sondennaher Konzentration) an der Strassenbrücke Hainburg vor allem bei höheren Durchflüssen gegen 1 bewegen.

Der Anstieg des Querprofilbeiwertes mit steigendem Durchfluss bedeutet jedoch, dass die Schwebstoff- konzentration in Ufernähe geringer ist als im Querprofil. Somit würde es in diesem Fall zu einer Überschätzung der Schwebstofffracht vor allem bei höheren Durchflüssen kommen. Davon ausgehend ist es unerlässlich, die räumliche Verteilung der Schwebstoffkonzentration über das Querprofil durch Messungen - vor allem bei höheren Durchflüssen - zu erfassen. Denn während dieser Hochwasserereignisse wird ein Großteil der Jahresfracht transportiert (Abb. 13a) (Haimann et al. 2014).

Die 5 Tage eines 15-jährlichen Ereignisses (03.-08.06.2010) und ein 10-jährliches Ereignis (14.-19.01.2011) hatten 


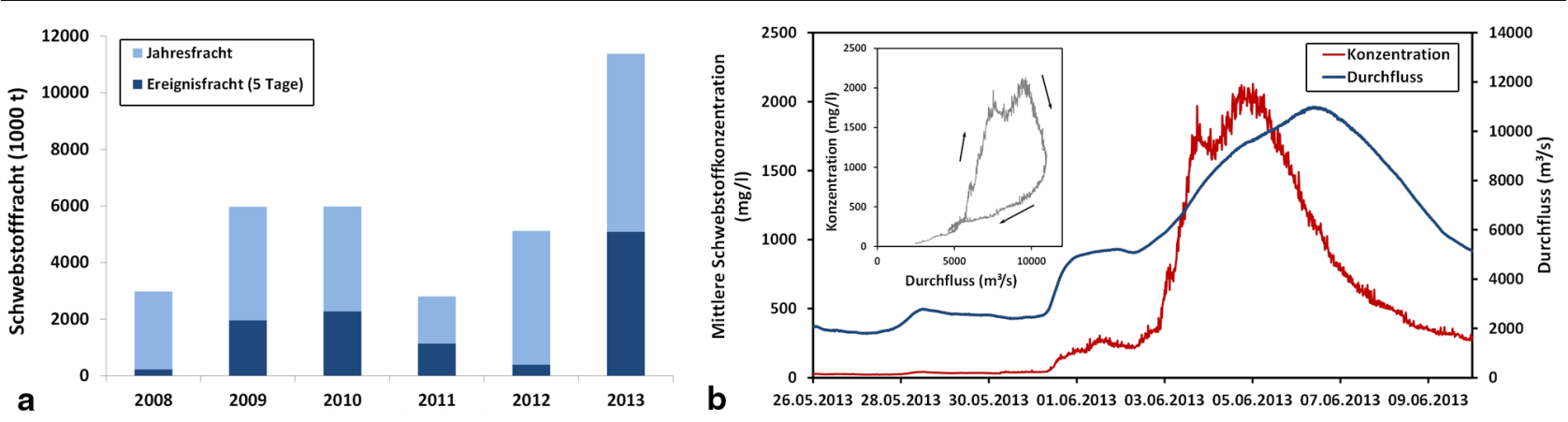

Abb. 13 a Schwebstofftransport Vergleich Jahres- und Ereignisfracht, b Schwebstoffsonden- und Durchflussganglinie HWEreignis 2013 für Hainburg Straßenbrücke - Insert: Hysterese-Effekt während des HW 2013

einen Anteil von 38 bzw. $41 \%$ an der gesamten Jahresfracht. Während des $\mathrm{HQ}_{200}$-Ereignisses vom 03.-08.06.2013) wurde annähernd gleich viel Schwebstoff transportiert wie im Jahr 2012 (4,9 Mio. t). Die Gegenüberstellung des Durchflusses und der mittleren Schwebstoffganglinie dieses Ereignisses bei der Hainburger Straßenbrücke (Abb. 13b) zeigen eine Hysterese, die Spitze der Trübung trat rund 2 Tage vor der eigentlichen Durchflussspitze auf, was Fragen hinsichtlich der Herkunft der Schwebstoffe aufwirft.

Betrachtet man die Anteile von Schwebstoff- und Geschiebejahresfracht an der Gesamtsedimentfracht, so entfallen 5 bis $12 \%$ des transportierten Sediments auf das Geschiebe. Dabei traten die 5\% im Jahr 2013 auf, in dem infolge des 200-jährlichen Ereignisses überdurchschnittlich viel Schwebstoff transportiert wurde. Im Mittel betrug der Anteil des Geschiebetransportes 8,8\%, exkludiert man das Jahr 2013 steigt der Geschiebeanteil auf $9,5 \%$.

\subsection{D Hydrodynamik, Sedimenttransport und Habitatmodellierung}

In der Pilotstrecke wurde eine Simulation der Hydrodynamik, des Sedimenttransportes und der Morphodynamik durchgeführt. Dabei gelang es, die in der Natur auftretenden Sohlformen auch numerisch zu modellieren (Tritthart et al. 2011). Voraussetzung für die erfolgreiche numerische Modellierung der räumlichen und zeitlichen Schwankungen der Sohlhöhe, des Geschiebetransportes bzw. der Korngrößen sind eine fraktionierte Berechnung inklusive Hiding/ExposureKorrektur, die fortlaufende Neusortierung des Sediments in einer Austauschschicht (active layer) sowie eine über die Fläche und Tiefe inhomogene Sediment- belegung. Darüber hinaus ist eine entsprechende Datengrundlage notwendig, um die empirischen Formeln im Modell kalibrieren zu können.

Die Habitatmodellierung ermöglicht eine Integration von abiotischen und biotischen Informationen, womit die Wirkung der Baumaßnahmen künftig im Postmonitoring beschrieben werden kann (vgl. Habersack et al. 2016, in dieser Ausgabe).

\section{Zusammenfassung und Schlussfolgerung}

Für die erfolgreiche Umsetzung innovativer Maßnahmen ist es einerseits wichtig, Werkzeuge zur Verfügung $\mathrm{zu}$ haben, um Auswirkungen und Veränderungen objektiv feststellen $\mathrm{zu}$ können, andererseits ist es aber auch erheblich, die Prozesse an sich verstehen zu lernen. Nur dadurch können Gründe für eine nicht erwartete Veränderung gefunden und letztlich Maßnahmen erfolgreich optimiert werden. Der Geschiebetransport an der Donau konnte durch die erste lange Reihe von Messdaten charakterisiert werden. Transportbeginn konnte schon bei Niederwasser festgestelltwerden, ab bordvollem Durchfluss stagniert der Geschiebetransportanstieg, bis er bei Extremereignissen wieder deutlich zunimmt. Der effektive Geschiebetransport (Durchfluss, der über die Zeit gesehen den größten Anteil am Geschiebetransport hat) konnte knapp über Mittelwasser gefunden werden. Die Tracersteine bewegten sich mehrheitlich uferparallel, bei einem Quertransport von $4 \%$ im Vergleich zum longitudinalen Transport. Hinsichtlich Bewegungshäufigkeit und Transportgeschwindigkeit zeigte sich ein größenselektives Verhalten, kleinere Steine bewegten sich häufiger, weiter und schneller im
Vergleich zu den mittleren und großen Steinen, welche eine ähnliche Transportcharakteristik aufwiesen. Der durchschnittliche Korndurchmesser bewegte sich vor Bau mit einer durchschnittlichen Geschwindigkeit von $3 \mathrm{~km} / \mathrm{Jahr}$. Direkt nach den Baumaßnahmen bewegten sich die Tracersteine etwas seltener und deutlich weniger weit als ein Stein während des Premonitorings. Eine weitere sehr wertvolle Methode zur Beurteilung von Stabilität und Einmischungsgrad der GSV war die Freeze-Core-Beprobung, die einen ungestörten Blick in die Donausohle ermöglicht. Kiesdünen bewegen sich an der Donausohle mit einer mittleren Geschwindigkeit von 5,6 m/h und bestimmen den Geschiebetransport maßgeblich. Ein Messgerät zur direkten Bestimmung der Sohlschubspannung an der Donausohle wurde entwickelt, um die zeitliche Fluktuation dieses Parameters aufzeichnen zu können, die letztlich der bestimmende Faktor für den Bewegungsbeginn des Sedimentes ist. Da selbst bei Hochwässern keine vollständige und gleichmäßige Durchmischung der Schwebstoffe im Profil auftritt, ist es unerlässlich, die Schwebstoffkonzentration auch über das Querprofil durch Messungen vor allem bei höheren Durchflüssen zu erfassen. Denn vor allem bei Hochwasserereignissen wird ein Großteil der Schwebstoffjahresfracht transportiert. Weiters trat während des $\mathrm{HQ}_{200}$ im Juni 2013 eine Hysterese bei der Schwebstoffsonde an der Hainburger Straßenbrücke auf. Denn die Spitze der Trübung trat rund 2 Tage vor der eigentlichen Durchflussspitze auf, was Fragen hinsichtlich der Herkunft der Schwebstoffe aufwirft. Eine 3D hydrodynamisch numerische Modellierung zeigt nun die Änderungen in der Sohlschubspannung auf und kann in Kombination mit einem Sedimenttransportmodell benutzt 
werden, um die Niederwasserregulierung zu optimieren, damit mit möglichst geringfügigen baulichen Anpassungen ein für alle Stakeholder positiver Zustand erreicht werden kann.

\section{Danksagung}

Die finanzielle Unterstützung durch das Bundesministerium für Wissenschaft, Forschung und Wirtschaft sowie die Nationalstiftung für Forschung, Technologie und Entwicklung wird dankend anerkannt. Die Autoren danken weiters viadonau, der Europäischen Union und dem Bundesministerium für Verkehr, Innovation und Technologie, die die Forschungsarbeit an der Donau mitfinanzieren, sowie den Gruppen FGP und Grundlagen von viadonau für die Zurverfügungstellung von Daten und die logistische Unterstützung bei Freilandmessungen. Die dargestellten Monitoringergebnisse wurden mit der Unterstützung von Margit Gerstl, Mario Klösch, Elisabeth Krinzinger, Silke Lackner, Martin Hinterleitner, Sebastian Pessenlehner und Márton Szinetár erzielt.

\section{Open Access}

Dieser Artikel unterliegt den Bedingungen der Creative Commons Attribution License. Dadurch sind die Nutzung, Verteilung und Reproduktion erlaubt, sofern der/die Originalautor/en und die Quelle angegeben sind.

\section{Einhaltung ethischer Richtlinien}

\section{Interessenkonflikt}

Es besteht kein Interessenkonflikt.

\section{Literatur}

Ancey, C., Heyman, J, (2014): A microstructural approach to bed load transport: mean behaviour and fluctuations of particle transport rates. J Fluid Mech 744: 129-168

Einstein, H. A., El Samni, E. A. (1949) Hydrodynamic forces on a rough wall. Rev Mod Phys 21: 520-524

Diplas, P., Dancey, C. L., Celik, A. O., Valyrakis, M., Greer, K., Akar, T. (2008): The role of impulse on the initiation of particle movement under turbulent flow conditions. Science 322(5902): 717-720

Ferreira, R. M. L., Hassan, M. A., Ferrer Boix C. F. (2015): Principles of bedload transport of non-cohesive sediment in open-channels. In Rowinsky P and Radecki-Pawlick A (Hrsg): Riversphysical, fluvial and environmental processes, 1. Aufl. Springer, Schweiz, S 323-373

Frey, P., Church, M. (2011): Bedload: A granula phenomenon. EARTH SURF PROC LAND 36: 58-69

Gmeiner, Ph., Liedermann, M., Tritthart M., Habersack, H. (2012): Development and testing of a device for direct bed shear stress measurement. In: Rutschmann P, Grünzner M, Hötzl S, Proceedings of the 2nd IAHR, Europe Conference, Munich, Germany, Jun 27-29, 2012
Grass, A. J. (1970): Initial instability of fine bed sand. J Hydr Div 96 (HY3): 619-632

Habersack, H., Hein, T., Liedermann, M. Bondar-Kunze, E. (2016): Erkenntnisse aus dem Pilotprojekt Bad Deutsch-Altenburg an der Donau für Schifffahrt, Ökologie und Wasserbau. Österreichische Wasser- und Abfallwirtschaft 68, H. $1-2, X X-X X$.

Haimann, M., Liedermann, M., Lalk, P., Habersack, H. (2014): An integrated suspended sediment transport monitoring and analysis concept. INT J SEDIMENT RES 29(2): 135-148

Liedermann, M., Tritthart, M., Habersack, $H$ (2013): Particle path characteristics at the large gravel-bed river Danube: results from a tracer study and numerical modelling. EARTH SURF PROC LAND 38(5): 512-522

Liedermann, M., Gmeiner, Ph., Tritthart, M. Glas, M., Habersack, H. (2014): Occurrence and characterization of bed forms at the Danube to the east of Vienna. In: CRC Press/Balkema, Proceedings of the International Conference on Fluvial Hydraulics, RIVER FLOW 2014: 1075-1080 Liedermann, M., Gmeiner, Ph., Glas, M. Tritthart, M., Habersack, H. (2016): Funktionalität der getesteten flussbaulichen Maßnahmen im Pilotprojekt Bad Deutsch-Altenburg. ÖWAW dieses Heft
McEwan, I, Heald, J. (2001): Discrete particle modeling of entrainment from flat uniformly sized sediment beds. Journal of Hydraulic Engineering 127 (7): 588-597

Milliman, J., Syvitski, J. (1992): Geomorphic tectonic control of sediment discharge to the ocean - the importance of small mountainous rivers. J Geol 100: 525-544

Shields, A. (1936): Anwendung der Ähnlichkeitsmechanik und der Turbulenzforschung auf die Geschiebebewegung. Mitteilungen der Preußischen Versuchsanstalt für Wasserbau 26, Berlin

Tritthart, M., Liedermann, M., Schober, B. Habersack, H. (2011): Non-uniformity and layering in sediment transport modelling 2: river application. J HYDRAUL RES 49(3): 335-344

Tritthart, M., Liedermann, M., Habersack, $H$. (2012): A meso-scale gravel tracer model for gravel-bed rivers. In: Murillo Munoz RE (Hrsg) River Flow 2012 Vol. 1, Taylor \& Francis, London, S 479-485

Zanke, U. (1990): Der Beginn der Geschiebebewegung als Wahrscheinlichkeitsproblem. Wasser und Boden 1 